\title{
RHEINZABERNER SIGILLATEN IN PANNONIEN
}

\author{
DÉNES GABLER
}

Forschungszentrum für Humanwissenschaften der Ungarischen Akademie der Wissenschaften Archäologisches Institut

Úri u. 49, H-1014 Budapest, Ungarn

gabler.denes@btk.mta.hu

\begin{abstract}
The sigillata of Rheinzabern are overrepresented among ceramic types in Pannonia at almost every archaeological site. Until today, from the excavated 266 sites, we know more than 15 thousand published Rheinzabern sigillata. At certain cases, this strikingly high ratio is a result caused by the creation of the given forts or settlements after $180 \mathrm{AD}$. At settlements that were supposedly abandoned already at the beginning of the 3rd century, the number of such ceramics is lower. The legionary fortress that provided most of the known Rheine goods is Vindobona, while the highest number of material concerning canabae was published from Aquincum. Among the urban communities, the quantity provided by the municipium of Brigetio holds the first place, while the utmost number of Rheinish ceramics among the villas was published from Baláca. At native settlements the quantity of imported samian is relatively low, as their inhabitants lacked the financial opportunities and also the needs for imported goods. At the sanctuary areas, almost three quarters of the material of Pfaffenberg in Carnuntum consists of sigillata of Rheinzabern, while in the Isis sanctuary in Savaria, this ratio is only 7,2\%. This type of sigillata is relatively rarely found in graves - we have 50 occurrences so far. Dishes intended for everyday usage of dwellers of towns and villas were also sigillata in many cases, while at settlements with native traditions they preferred bowls with reliefs. Sigillata of Rheinzabern emerged in Pannonia already in the Antonine age, but at this time yet they were underrepresented in the presence of the sigillata of Lezoux. The greatest amount of Rheinzabern was transported into the province in the middle production section of the manufactory, i.e., between 180 and 230, in the age of the Severan conjuncture. Material dated to the middle third of the 3rd century in Pannonia is insignificant $(2 \%)$, which can be attributed to the stagnation of trade relations, and to the inner and outer political situation of the Empire.
\end{abstract}

Keywords: Samian ware, Rheinzabern sigillata, settlements, urban communities sanctuary, Pannonian cemeteries

Mit Ausnahme einiger Fundorte macht die Rheinzaberner Ware überall den größten Anteil des Fundmaterials in Pannonien aus: Von den Produktionsstätten wurden Sigillaten in der größten Menge von hier nach Pannonien geliefert. Auf mehreren Fundorten ist die Menge der aus dieser rheinischen Manufaktur stammenden Sigillaten beträchtlicher als die aus allen anderen Manufakturen insgesamt. Von wenigen Ausnahmen abgesehen vertreten die rheinischen Sigillaten den größten Prozentsatz und die größte Menge auf den gegebenen Fundorten (Abb. 1).

Brigetio-municipium $^{1}$

Carnuntum-Pfaffenberg mons sacer

Aquincum-Zivilstadt

Ulcisia Castra-Auxiliarkastell

Poetovio-colonia

Visegrád-Lepence Siedlung

${ }^{1}$ T. BECK: Terra Sigillata aus Brigetio. Vorbericht zu den Funden der Grabungen 1992 bis 1996. Die autonomen Städte in Nori-
$45,4 \%$

$73,8 \%$

$44,8 \%$

$73,5 \%$

$44,1 \%$

$62,3 \%$

cum und Pannonien. Hrsg. von M. Šašel-Kos und P. Scherrer. Situla 42. Ljubljana 2004, 247. 
Vindobona-Legionslager

Baláca-Villa und canabae legionis

Páty - einheimische Siedlung

Aquincum-canabae legionis

Gorsium $^{2}$

Budaörs - einheimische Siedlung (vicus)

Carnuntum-Zivilstadt

Aquincum-Wasserstadt (Alenkastell und Siedlung) ${ }^{3}$

Brigetio-municipium (2011)

Strebersdorf-Siedlung 5

Sárvár-Straßenstation
$61,8 \%$

$39,5 \%$

$60,7 \%$

$39,0 \%$

$56,9 \%$

$53,0 \%$

$39,0 \%$

$36,4 \%$

$52,5 \%$

$51,0 \%$

$33,0 \%$

In der Provinz kennen wir 14500 Rheinzaberner Sigillaten von 266 Fundorten. ${ }^{6}$ Das bedeutet zugleich, dass weiße Lücken auf der Karte praktisch nur in den ununtersuchten Gebieten zu beobachten sind (Abb. 2). Diese Menge setzt sich überwiegend aus den veröffentlichten Fundmaterialien zusammen. Aber das bedeutet bei Weitem nicht, dass alle Exemplare in jedem Fall einzeln publiziert wären. Manchmal wird es nur aus den statistischen Ta-

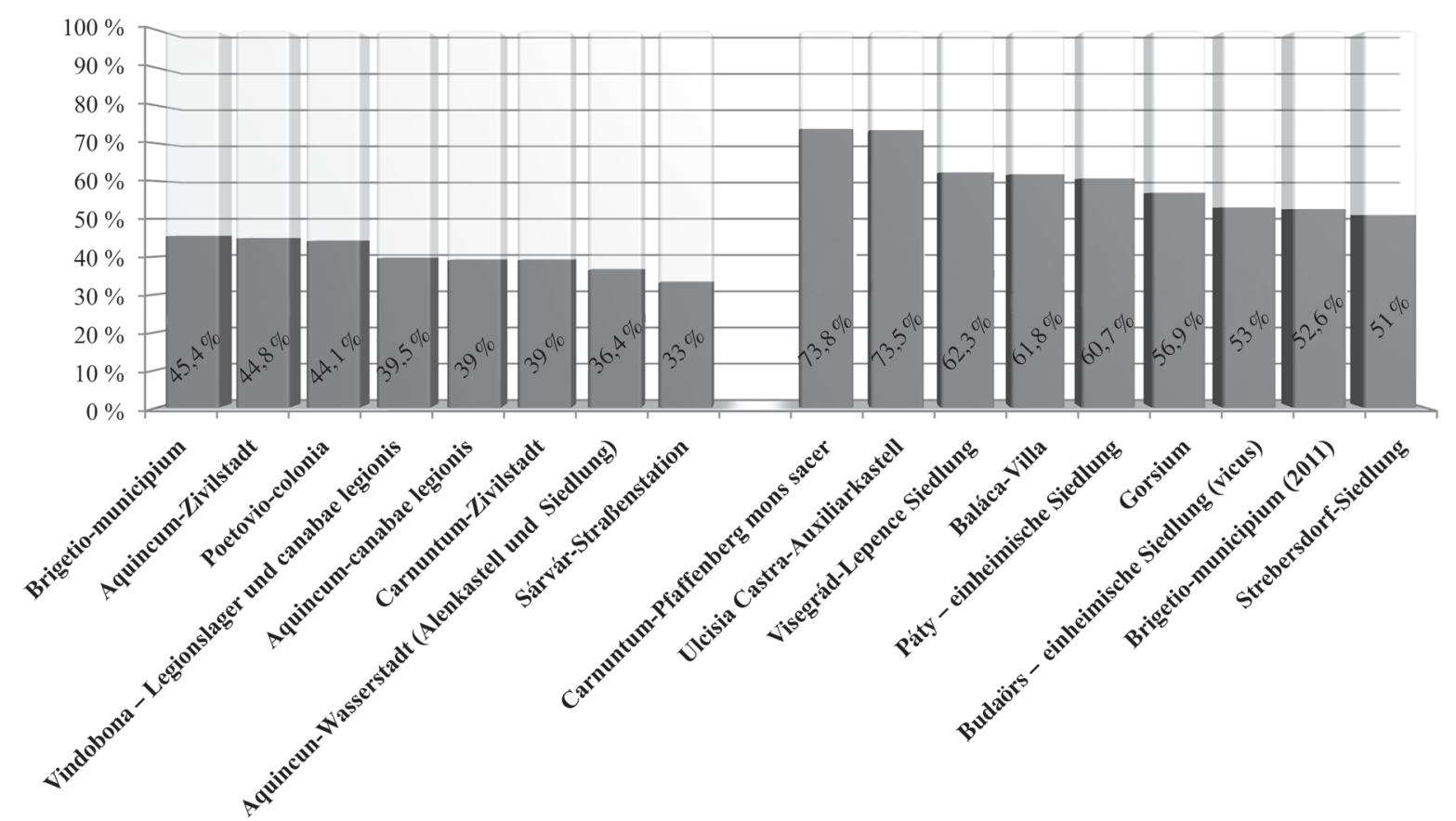

Abb. 1. Prozentuelle Anteilnahme der Rheinzaberner Sigillaten in verschiedenen Fundorten Pannoniens

${ }^{2}$ É. KoczTUR: Ausgrabungen im südlichen Stadtviertel von Gorsium (Tác-Margittelep). Alba Regia 13 (1972) 69-149; Zs. BÁNKI: Forschungen in Gorsium im Jahre 1976. Alba Regia 17 (1979) 205-243.

${ }^{3}$ D. GABLER: Terra sigillaták Aquincum legkorábbi táborából és annak helyén emelt későbbi római épületekből (Samian ware from the earliest fort of Aquincum). Budapest Régiségei 45 (2012) 123-126, Abb. 6.

${ }^{4}$ D. Bartus-L. Borhy-G. Delbó-K. Dévai-Z. KisA. NAGY-K. SEY-E. SZÁMAdó-Zs. SZÓRÁdI-I. VIdA: Jelentés a KoActa Archaeologica Academiae Scientiarum Hungaricae 67, 2016
márom/Szőny-Vásártéren 2011-ben folytatott régészeti feltárások eredményeiről (Bericht über die Ergebnisse der im Jahre 2011 in Brigetio (Fo. Komárom/Szőny-Vásártér) geführten archäologischen Ausgrabungen). Kuny Domokos Múzeum Közleményei 19 (2014) 27-56. ${ }^{5}$ J. MATOUSCHEK-H. NowAK: Fundchronik. Strebersdorf. Fundberichte aus Österreich 37 (1998) 755-772.

${ }^{6}$ Über die Verbreitung der Rheinzaberner Sigillaten in Pannonien erschien zuletzt: D. GABLER: Einige Besonderheiten der Verbreitung der Rheinzaberner Sigillaten in Pannonien. BVbl 52 (1987) 75-104. 


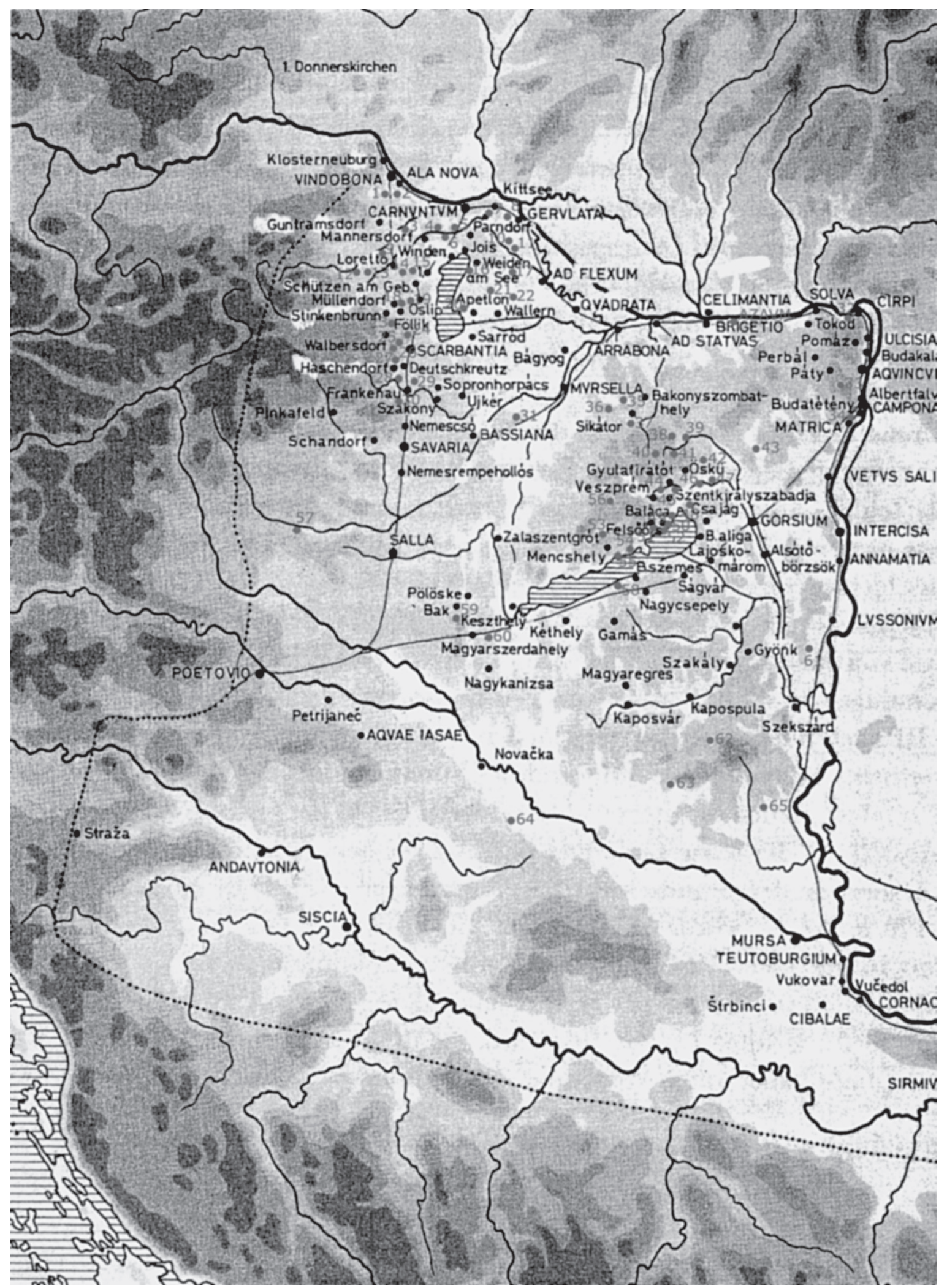

Abb. 2. Fundorte der Rheinzaberner Sigillaten in Pannonien (s. s. 125-126) 
bellen klar, wie viel Stücke die rheinische Manufaktur vertreten. Obzwar die Rheinzaberner Sigillaten beinahe überall aufzufinden sind, können wesentliche Unterschiede bei der Verteilung beobachtet werden. Im Nachstehenden werden diese Abweichungen vorgestellt. Die herausragend hohen Prozentwerte können bei Ulcisia Castra dadurch erklärt werden, dass das Auxiliarlager, in dessen Gebiet die Freilegungen durchgeführt wurden, wahrscheinlich erst nach $180 \mathrm{n}$. Chr. ausgebaut wurde. Das frühere Kastell könnte woanders gestanden haben. Auch auf dem Pfaffenberg bei Carnuntum kann die wichtigste Periode der Kulttätigkeit nach 180 datiert werden. ${ }^{7}$ Wesentlich niedriger als der Durchschnitt (33-45\%) ist der Anteil der Rheinzaberner Sigillaten im Lager und im vicus von Albertfalva ${ }^{8}$ (kaum $10 \%$ ), die nach den Markomannenkriegen von 166 bis $180 \mathrm{n}$. Chr. wahrscheinlich aufgelassen wurden (Abb. 3). Auffallend niedrig ist der Anteil der rheinischen Sigillaten im Fundmaterial des Iseum von $\operatorname{Savaria}^{9}(7,2 \%)$ - trotzdem, dass die Blütezeit des Heiligtums eben in die Severerzeit zu datieren ist. Auch auf dem südlichen Teil der canabae von Aquincum kamen wenige Sigillaten zum Vorschein ${ }^{10}$ (19 und 9,3\%), was dadurch zu erklären ist, dass dieser Siedlungsteil am Anfang des 3. Jahrhunderts teils aufgegeben wurde. Zugleich ist dieser Anteil auf dem nördlichen Teil der canabae, wo sich die Amtsgebäude der Statthalterschaft befanden bzw. hochrangige Beamter und Offiziere im 3. Jahrhundert angesiedelt wurden, 64,7 \%. Nur im Fundmaterial des Auxiliarlagers und des vicus von Gerulata ist der Anteil der Rheinzaberner Sigillaten niedriger als der Durchschnitt (23,3\%) und das kann unter den Fundorten entlang des Limes als ein Ausnahmefall betrachtet werden. ${ }^{11}$

Von den Legionslagern sind die meisten rheinischen Waren bis dahin in dem von Vindobona bekannt: Von I. Weber-Hiden wurden 1028 reliefverzierte Sigillaten von 17 Fundorten veröffentlicht. ${ }^{12}$ Viel wenigere Exemplare kamen im Legionslager von Carnuntum ans Tageslicht (etwa 100 St.), während die Stückzahl im Legionslager von Brigetio und Aquincum nicht einmal annähernd zu bestimmen ist. Da der genaue Fundort in der Veröffentlichung der Sigillaten weder von Brigetio noch von Aquincum angegeben wurde, können sowohl die canabae als auch die Zivilstadt im Fall der von Gy. Juhász publizierten Sigillaten als Fundstellen in Frage kommen. ${ }^{13}$ Noch dazu ist es manchmal auch nicht sicher, ob alle in den Sammlungen aufbewahrten Stücke aus Brigetio stammen.

Von den canabae an den Legionslagern kann man mit rheinischen Gefäßen in größerer Menge in Vindobona rechnen. ${ }^{14}$ Von 16 Fundorten sind 626 Gefäße bekannt, während 710 Exemplare von neun Fundorten im Gebiet der canabae von Aquincum veröffentlicht wurden. ${ }^{15}$

Von den Funden der Auxiliarlager wurden die meisten Stücke bis jetzt aus Carnuntum veröffentlicht (625 St.), ${ }^{16}$ ferner in Gerulata bzw. Ad Statuas kennt man 100 bzw. 170 Rheinzaberner Sigillaten. Dank der Freilegungen von 2001 bis 2004 kamen 1010 rheinische Sigillaten im Auxiliarlager von Ulcisia Castra (Szentendre) ans Tageslicht.

${ }^{7}$ I. PISO: Inschriften. Das Heiligtum des Jupiter Optimus Maximus auf dem Pfaffenberg Carnuntum 1. RLÖ 41. Wien 2003.

${ }^{8}$ D. GABLER: Terra sigillaták az albertfalvai vicus nyugati részén (Terra Sigillaten im Westteil des Vicus von Albertfalva). Budapest Régiségei 40 (2006) 71-93.

${ }^{9}$ D. GABLER: Le questioni cronologiche del tempio di Iside a Savaria. In: Proceedings of the 2nd International Conference on the Danubian Provinces, Ferrara 20-22th Nov. 2013. Ed. by L. Zerbini and A. Andreoli. Bologna 2015, 254.

${ }^{10}$ P. VÁmos: Fazekasműhely az aquincumi canabae déli részén (Töpferwerkstätte im südlichen Teil der canabae legionis von Aquincum). Archaeologiai Értesítő 127 (2002) 57-59, 67, 75; J. LEBEGYEV-A. MÁRTON: Újabb terra sigillata leletek az aquincumi katonavárosi canabae területéről (New Samian Ware Finds from the canabae legionis of Aquincum). Archaeologiai Értesítő 128 (2003) 134, 149-153, 167.

${ }^{11}$ D. Gabler-M. Pichlerová: Terra Sigillaten von Gerulata. In: Gerulata I. Hrsg. von K. Kuzová und J. Rajtár. Arch. Inst. der Slowakischen Akademie. Nitra 1996, 64-69, 78, 80-83, 97104; J. DeKan-K. KuZmová: Terra sigillata in Rusovce-Bergl reperta annis 1964-1969. In: Gerulata I. Nitra 1996, 161-163, 168-169; E. KREKOVIĊ: Terra sigillata aus Grabungen und Lesefunden in Rusovce-Bergl und seiner nächsten Umgebung (1976-1978). In: Gerulata I. Nitra 1996, 195-196, 202; V. VARSIK-K. KUZMOVÁJ. SchmidtovÁ: Archäologische Untersuchungen bei der Bautätig-

Acta Archaeologica Academiae Scientiarum Hungaricae 67, 2016 keit in Rusovce. In: Gerulata I. Nitra 1996, 217-218, 220, 223, 225-226.

${ }^{12}$ I. WEBER-Hiden: Die reliefverzierte Terra Sigillata aus Vindobona. Teil 1. Legionslager und Canabae. Wiener Arch. Studien 1. Wien 1996, 210-341, Taf. 81-145.

${ }^{13}$ GY. JuHÁsZ: A brigetioi terra sigillaták - Die Sigillaten von Brigetio. Dissertationes Pannonicae Ser. 2. No. 3. Budapest 1935, 46-115; K. KuZMovÁ: Terra sigillata in der Museumssammlung. Podunajské Múzeum Katalog III. Rímske zbierky. Komarno 1992, 84-85.

${ }^{14}$ I. WeBER-Hiden 1996, 20; P. DonAT: Terra sigillata vom Michaelerplatz. Fundort Wien 2 (1999) 213.

${ }^{15}$ D. GABLER: Terra Sigillaten in einem Luxusgebäude im nördlichen Teil der canabae von Aquincum (Budapest III. Bez., Folyamör Str.). Schriften zur Archäologie und Archäometrie der Paris Lodron Universität Salzburg 7. Ein kräftiges Halali aus der Römerzeit. Norbert Heger zum 75. Geburtstag. Hrsg. von F. Lang, St. Traxler, E. M. Ruprechtsberger und W. Wohlmayr. Salzburg 2014, 76-80.

${ }^{16}$ CH. KAndLeR-Zöchmann: Terra Sigillata der Grabungskampagne 1993. In: M. Kandler (Hrsg.): Das Auxiliarkastell Carnuntum 2. Forschungen seit 1989. Sonderschriften des Österreichischen Arch. Instituts 30. Wien 1997, 101-171, besonders 106-108, Taf. 21-25; M. KRONBERGER: Das Auxiliarkastell Carnuntum 1. Forschungen 1977-1988. Hrsg. von H. Stiglitz und S. Jilek. Sonderschriften des Österreichischen Arch. Instituts 29. Wien 1997, Taf. 12, 77-135, $112-117$. 


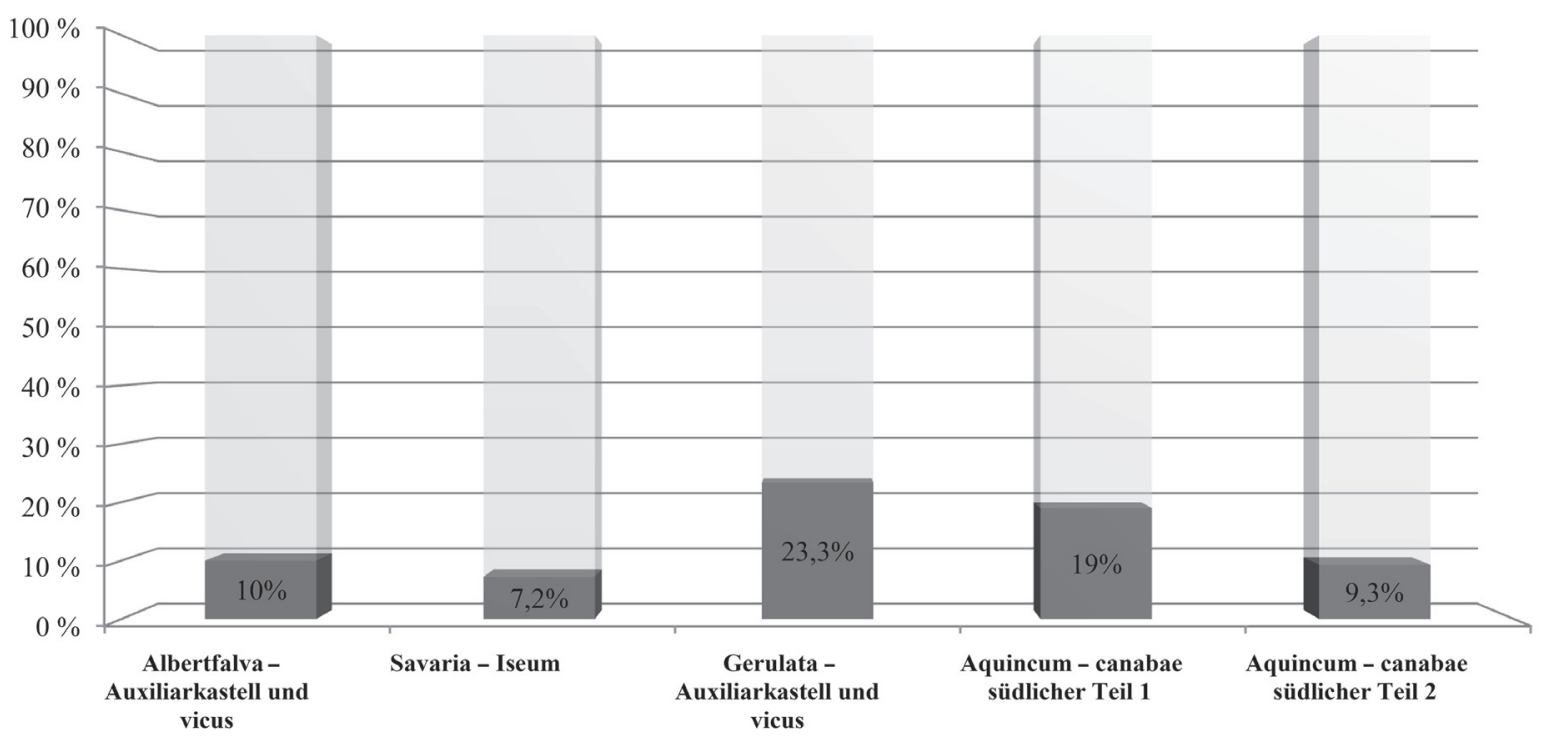

Abb. 3. Prozentuelle Anteilnahme der Rheinzaberner Sigillaten in Fundorten mit niedriger Anteilnahme

Von den Munizipien (municipia, coloniae), die zumeist neben militärischen Lagern entstanden (Abb.4), sind die meisten Exemplare in Brigetio bekannt (1400 St.). Das Fundmaterial der hiesigen, seit 1992 durchgeführten Ausgrabungen wurde in mehreren Publikationen vorgeführt. In der Zivilstadt von Aquincum sind 911 Rheinzaberner Gefäße von sechs Fundorten bekannt, während 472 rheinische Sigillaten aus Poetovio, 316 Stücke aus Carnuntum ${ }^{17}$ und 304 Stücke aus Cibalae publiziert wurden. ${ }^{18}$ Wird das Fundmaterial der Städte untersucht, dann findet man relativ wenige rheinische Sigillaten in Vindobona (Rennweg $44-19,2 \%$ ), Salla $(23 \%)^{19}$ und Siscia $(14 \%){ }^{20}$

Aus dem Auxiliarvicus Azaum wurden 101 Sigillaten (32,23\%) bekannt gemacht. ${ }^{21}$

Von den Villenwirtschaften sind die meisten rheinischen Gefäße bis jetzt in Baláca publiziert ${ }^{22}$ (454 St. 61,8\%). An anderen Fundorten wird diese Manufaktur nur durch einige Dutzend Exemplare vertreten (Szentkirályszabadja-Romkút 42 St., Gyulafirátót-Pogánytelek 16 St., ${ }^{23}$ St. Martin a. d. Raab ${ }^{24} 14$ St.). Insgesamt in 59 Siedlungen kamen Rheinzaberner Sigillaten zum Vorschein. Die überwiegende Mehrheit wurde im westlichen Teil der Provinz, im heutigen Burgenland, gefunden. Die meisten Stücke sind Lesefunde, ihre Veröffentlichung macht doch möglich, über die Bedeutung des rheinischen Imports ein besseres Bild zu bekommen.

$\begin{array}{lr}\text { Visegrád-Lepence Siedlung } & \\ \text { Budaörs } & 644 \text { St. }(62,3 \%) \\ \text { Páty } & 505 \text { St. }(53,0 \%) \\ \text { Szakály-Réti földek } & \text { 295 St. }(60,7 \%) \\ \text { Strebersdorf } & 88 \text { St. }(73,9 \%) \\ & 61 \text { St. }\end{array}$

${ }^{17}$ A. RaUCHENWALD: Die Funde der Ausgrabungen 19861990 in der Zivilstadt Carnuntum-Insula VI. Carnuntum Jahrbuch 1996, 71, 74-79, 108-120, 130-132, 140-144, 148-150, 154-164.

${ }^{18}$ T. LeleKović: Relief terra sigillata from Cibalae (Vinkovci, Croatia): A typology. Acta RCRF 40 (2008) 179-181.

${ }^{19}$ D. GableR: Terra sigillata. In: F. Redő (Hrsg.): Kereskedők Sallában - Commercianti di Salla. Hereditas Sallensis 3. Zalalövö 2005, 42-45.

${ }^{20}$ R. MAKJanić: Terra sigillata. In: R. Koščeviċ-R. Makjanic: Siscia. Pannonia Superior. Finds and Metalwork Production. BAR Int. Ser. 621 (Oxford 1995) 65-70.

${ }^{21}$ F. HORVÁtH: Terra sigillata aus dem SW-Kastellvicus Azaum/Odiavum aus den Jahren 1998-2000. Pannonica. Provincialia

et Archaeologia. Studia sollemnia auctorum Hungarorum Eugenio Fitz octogenario dedicata. Libelli archaeologici ser. nova 1. Budapest 2003, 139-196.

${ }^{22}$ D. GABLER: Die Sigillaten von Baláca 4. Balácai Közlemények 7 (2002) 69-108.

${ }^{23}$ O. CSIRKe-D. Gabler-S. PALÁGyi: Terra sigillata edények a veszprémi Laczkó Dezső Múzeum gyüjteményében (Terra Sigillata-Gefäße des Museums „Laczkó Dezso““ in Veszprém). Veszprém 2006, 172-177, 189-196.

${ }^{24}$ S. LAMM: St. Martin a. d. Raab. Fundberichte aus Österreich 45 (2006) 397.

${ }^{25}$ Grabung von P. Gróf im Jahr 2008. Unveröffentlicht. 


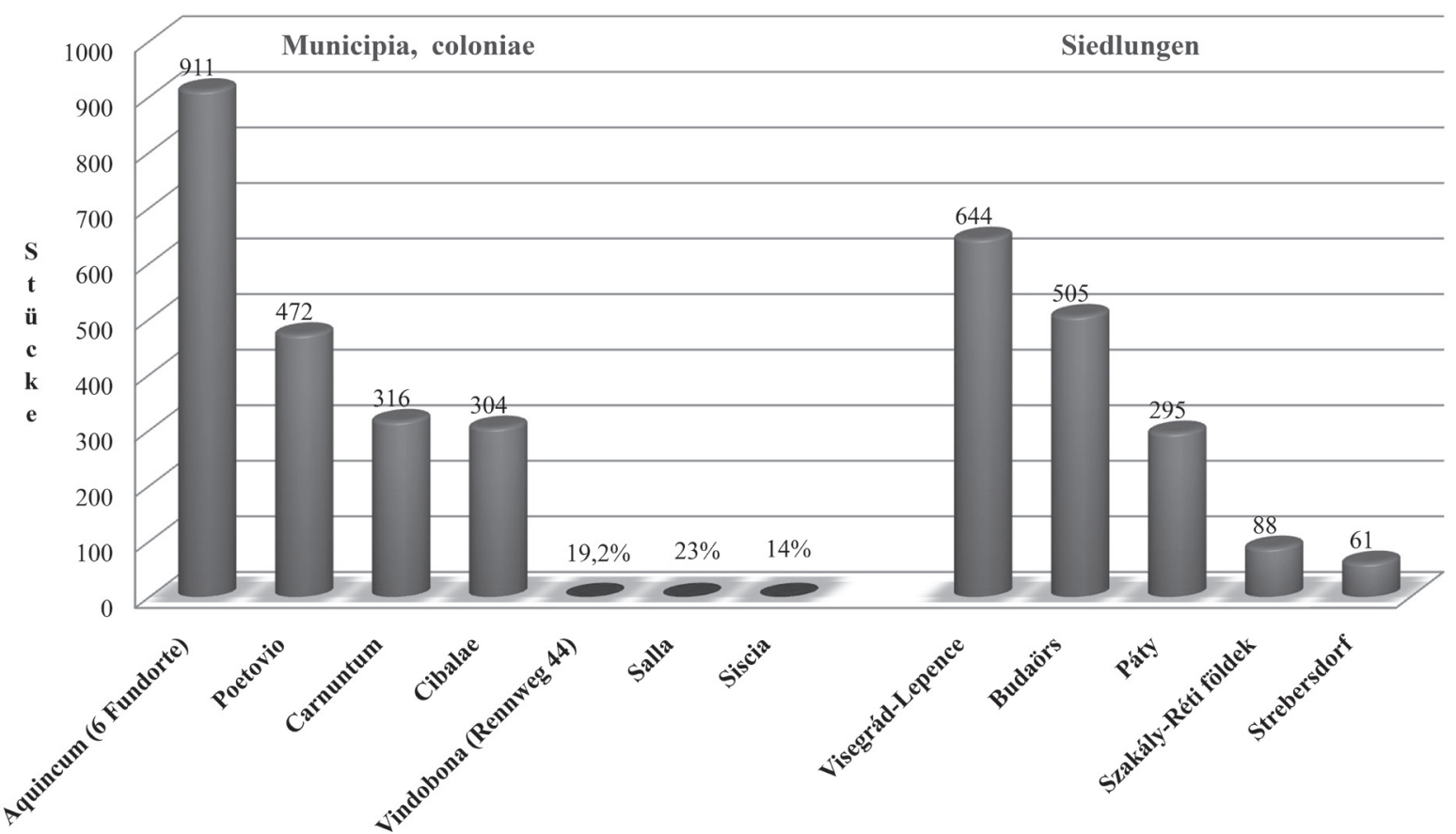

Abb. 4. Anteil der Rheinzaberner Sigillaten in den Städten und in den dörflichen Siedlungen Pannoniens

Von den oben erwähnten sind die Siedlungen von Páty, ${ }^{26}$ Budaörs und Szakály ${ }^{27}$ seit der LT D-Periode fortlebende einheimische Siedlungen. Das in der Nähe von Aquincum befindliche, ausgedehnte vicus von Budaörs ${ }^{28}$ ausgenommen kamen relativ wenige Importkeramik in diesen Siedlungen ans Tageslicht, da die finanzielle Lage der Bewohner es nicht möglich machte, Importwaren dieser Art, auf die sie auch sonst keinen Anspruch hatten, zu verschaffen. Diese Siedlungen lagen im Allgemeinen außerhalb des Netzes der wichtigen Handelsstraßen.

Unter den Fundorten können zwei größere Heiligtumsbezirke erwähnt werden, in denen die rheinische Ware in bedeutender Menge zum Vorschein kam. Einer der Heiligtumsbezirke wurde auf dem Pfaffenberg bei Carnuntum ausgebaut. Da wurden relativ wenige Sigillaten gefunden (343 St.), aber 73,8 \% der Funde war Rheinzaberner Ware. ${ }^{29}$ Im Areal des Isis-Heiligtums von Savaria, das so groß wie eine insula ist, ist die Zahl der bis dahin bestimmten Gefäße fünfmal so groß, aber nur 7,2 \% der Funde ist rheinische Ware. Das ist überraschend, weil der bedeutendste Umbau - d. h. Erweiterung - des Heiligtumsbezirkes aufgrund der Inschriften eben in die severische Zeit zu datieren ist. Man könnte vielleicht auch an die Besonderheiten des Kultes oder an die Änderung der Opfergabe denken, aber im 3. Jahrhundert nimmt nicht nur die Menge der Importwaren, sondern auch der Geldumlauf ab. In Savaria selbst, in der Stadt sind sonst die Rheinzaberner Sigillaten in bedeutenderer Menge aufzufinden. ${ }^{30}$

An den Straßenstationen kommen im Allgemeinen wenige Sigillaten ans Tageslicht, obwohl der Ausbau und das Bestehen der Straßenstationen an das Endes des 2. und in die erste Hälfte des 3. Jahrhunderts datierbar sind

${ }^{26}$ D. GABLER: Terra sigillaták a pátyi telepen. Studia Comitatensia 30 (2007) 239-270.

${ }^{27}$ D. GABLER-F. HoRvÁth: A szakályi terra sigillaták és helyük a bennszülött telep kerámiaspektrumában (Die Terra Sigillaten von Szakály und ihre Stellung im Keramikspektrum der einheimischen Siedlung). A Wosinsky Mór Múzeum Évkönyve 19 (1996) 148-150, Abb. 4-8.

${ }^{28}$ D. GABLER: Terra sigillaták. In: K. Ottományi (Hrsg.): Római vicus Budaörsön (Der römische Vicus in Budaörs). Budapest 2012, 422-435.
${ }^{29}$ Aufgrund der Bestimmungen von I. Koós in: D. GABLER: Terra sigillata auf dem Pfaffenberg. Manuskript 2005.

${ }^{30}$ Sz. Bíró: Savaria nyugati temetője (Das westliche Gräberfeld von Savaria). Savaria 28 (2004) 63-134; E. N. FÜLöP: Terra sigillata leletek Savariából, a Perint patak nyugati oldaláról (Terra Sigillata-Befunde aus Savaria an/von der westlichen Seite des Perint Baches). Savaria 28 (2004) 139-158. 
(Nemescsó, Sorokpolány, Biedermannsdorf). ${ }^{31}$ Viele Rheinzaberner Sigillaten kamen in der Nähe der Straßenstation von Sárvár zum Vorschein, aber die meisten Stücke sind in Privatsammlungen zu finden. ${ }^{32}$ So ist es gar nicht sicher, dass sie an der Straßenstation, an der Übergangsstelle über die Raab gefunden wurden.

Bis jetzt kamen 53 Exemplare in Gräberfeldern an der ripa (Mursa, Intercisa, Aquincum, Ulcisia Castra), ferner an der Bernsteinstraße ans Tageslicht. Von ihnen sind nur drei Exemplare reliefverziert, die anderen sind Teller der Form Drag. 31, Dr. 32 und Lud Tb bzw. Becher der Form Drag. 33 und Drag. 54. Allein im Grab 78 von Carnuntum kamen fünf rheinische Sigillaten zum Vorschein; alle vertreten die glatte Ware. In den Gräberfeldern von Carnuntum sind 18, in Gerulata sechs, Intercisa elf, Matrica elf, Söjtör vier und Poetovio drei Stücke bekannt. ${ }^{33}$ Aus Vindobona veröffentlichte I. Weber-Hiden zahlreiche Exemplare von 16 Fundorten, aber sie macht darauf aufmerksam, dass es sich hier allem Anschein nach um Bereiche handelt, in denen sich canabae und Gräber verflechteten oder gar überlagen. Was sich dort wirklich abgespielt hatte, wird wohl erst dann zu erkennen sein, wenn es gelungen ist, die Grabensembles wieder zusammenzustellen und zu datieren. Canabae und Gräberfelder sind nicht eindeutig voneinander zu trennen. ${ }^{34}$

Wo die Untersuchung überhaupt möglich war, zeigt die Verteilung des Rheinzaberner Fundmaterials ein abwechslungsreiches Bild. In den Städten kommt die glatte Ware in bedeutend größerer Zahl als die reliefverzierte $\operatorname{vor}(A b b .5)$.

\begin{tabular}{|c|c|c|c|c|}
\hline Brigetio-municipium & \multicolumn{2}{|c|}{190 Bilderschüsseln } & \multicolumn{2}{|c|}{430 glatte Ware } \\
\hline Aquincum-Zivilstadt & 284 & $»$ & 544 & $"$ \\
\hline Aquincum-canabae & 136 &, & 205 &, \\
\hline Carnuntum-Steilhang ${ }^{35}$ & 78 & , & 213 & , \\
\hline
\end{tabular}

Bei den Legionslagern kann dieses Verhältnis nicht untersucht werden. Aus Vindobona wurden bisher nur die Bilderschüsseln veröffentlicht und ausgenommen das Legionslager von Carnuntum kann das Fundmaterial des Legionslagers und der canabae bei den anderen nicht abgesondert werden.

Bei den Auxiliarlagern ist schon eine andere Verteilung zu beobachten.

\begin{tabular}{|c|c|c|}
\hline Carnuntum & 226 Bilderschüsseln & 266 \\
\hline Ad Statuas & $40 \quad$ & 62 \\
\hline Azaum & 50 & 51 \\
\hline Gerulata & 65 & 66 \\
\hline
\end{tabular}

Da ist also der Anteil der reliefverzierten und glatten Gefäße gleich. Auch bei den Villenwirtschaften macht die unverzierte Ware die Mehrheit aus.

$\begin{array}{lcc}\text { Baláca-Herrensitz } & 201 \text { Bilderschüsseln } & 253 \text { glatte Ware } \\ \text { Baláca }- \text { Gebäude } \mathrm{II}^{36} & 40 \quad \# & 59 \%\end{array}$

Ein ganz abweichendes Bild zeigt das Fundmaterial der Siedlungen der Eingeborenen. Da ist die überwiegende Mehrheit der Sigillaten reliefverziert.

$\begin{array}{lcc}\text { Budaörs } & 386 \text { Bilderschüsseln } & 119 \text { glatte Ware } \\ \text { Szakály } & 68 \% " & 20 \%\end{array}$

${ }^{31}$ S. Groh-H. Sedlmayer-Cs. V. ZalKa: Die Straßenstationen von Nemescsó und Sorokpolány an der Bernsteinstraße (Pannonien, Ungarn). Zentraleuropäische Archäologie 3. Wien 2013, 30, 37, 95-102.

${ }^{32}$ F. HORVÁTH: Terra sigillata-magángyüjtemény Sárvár és környékéről (Eine Terra Sigillata-Privatsammlung aus Sárvár und Umgebung). Communicationes Archaeologicae Hungariae 2001, 90, $114-120$.

\footnotetext{
${ }^{33}$ A. MÁrTon: Mobilier en terre sigillée dans les sépultures pannoniennes. Communicationes Archaeologicae Hungariae 2008, 135-189, bes. 143 .

${ }^{34}$ WeBER-Hiden 1996, 15.

${ }^{35}$ CH. Kandler-Zöchmann: Terra Sigillata der ,Steilhang-Grabung“ 1971-1972 in Petronell. Carnuntum Jahrbuch 2001 (2002) 79-92.

${ }^{36}$ D. GABLER: A balácai II. épület sigillatái (Sigillaten aus dem Gebäude II aus Baláca). Balácai Közlemények 8 (2004) 123-163.
}

Acta Archaeologica Academiae Scientiarum Hungaricae 67, 2016 
Das bedeutet so viel, dass auch die alläglich benutzten Teller und Becher der Städte- und Villenbewohner Sigillatagefäße waren, während man nur die „Bilderschüsseln“ in den Siedlungen mit einheimischer Tradition gern hatte. Eine ähnliche Beobachtung konnte auch in dem mit Pannonien benachbarten Barbaricum gemacht werden, wo dieser Anteil für die reliefverzierten Gefäße noch auffälliger ist (78\%). ${ }^{37}$

Die Analyse der Bilderschüsseln zeigt, dass Rheinzabern von der Mitte des 2. Jahrhunderts an ${ }^{38}$ auf den Märkten der Donauprovinzen ein Rivale der Manufaktur von Lezoux und der anderen mittel- und ostgallischen Töpfereien war. ${ }^{39}$ Früher haben wir schon untersucht, wie hoch der Anteil Rheinzaberns in der Zeit des Höhepunkts des Imports aus Lezoux in den Jahrzehnten vor den Markomannenkriegen in Pannonien war. ${ }^{40}$ In den Fundkomplexen, die mit den Ereignissen der Markomannenkriege in Verbindung gebracht werden können, kamen Rheinzaberner Sigillaten nur in ganz geringer Anzahl vor. Alle Stücke des Depots von Gorsium (verborgen im Jahre 178 n. Chr.) sind mittelgallische Gefäße, ${ }^{41}$ genau wie im Auxiliarkastell von Arrabona. ${ }^{42}$ Im Bestand des sog. Geschirrladens von Aquincum-Zivilstadt überwog die Ware aus Lezoux in so hohem Maße, dass Gy. Juhász diesen Komplex für eine Niederlassung der Manufaktur Lezoux in Aquincum halten konnte, ${ }^{43}$ zumal die Verfasserin die Gefäße des Cobnertus unbegründet für Ware des Töpfers von Lezoux hielt. Unter den 55 von ihr publizierten Sigillaten können 51 Lezoux zugewiesen und nur vier mit dem Töpferzentrum von Rheinzabern in Verbindung gebracht werden. In den Auxiliarkastellen tauchen auch Rheinzaberner Bilderschüsseln in den antoninischen Brandschichten auf.

\section{Januarius I \\ Reginus I \\ Cerialis V}

\author{
Vetus Salina ${ }^{44}$ \\ Ad Statuas ${ }^{45}$ \\ Gerulata, Quadrata, Intercisa ${ }^{46}$
}

Exemplare der letztgenannten Ware wurden auch in Salla gefunden. Diese Stadt war wahrscheinlich durch die Markomannen im Jahre 170 n. Chr. im Laufe des Angriffs gegen Aquileia zerstört. Durch die Funde lassen sich Schlüsse über den Einfluss des Krieges auf das Wirtschaftsleben und die Handelsbeziehungen schließen. Es scheint allerdings, als ob das näher liegende Rheinzabern den verkehrsgeographischen Vorteil und die Möglichkeiten, die sich in den friedlichen Übergangsperioden boten, besser ausnutzen könnte als Lezoux. Aus diesem Grund tauchen die mittelgallischen Sigillaten nach 175/178 in viel geringerer Anzahl in den Donauprovinzen als in Britannien auf. In Raetien endete der Import der mittelgallischen Sigillaten ganz abrupt mit dem Ende der Markomannenkriege, d. h. mit dem Beginn des Legionslagers von Regensburg, also um 180 n. Chr. ${ }^{47}$

${ }^{37}$ D. GABLER: Terra sigillaták Cegléd és Abony határából [Samian ware from Cegléd and Abony]. Studia Comitatensia 31 (2011) 262.

${ }^{38}$ A. MEEs: Organisationsformen römischer Töpfermanufakturen am Beispiel von Arezzo und Rheinzabern mit Berücksichtigung von Papyri, Inschriften und Rechtsquellen. Röm.-Germ. Zentralmuseum Monographien. Mainz 2002, 78-79; H.-G. SIMON in: D. BAATZ: Hesselbach und andere Forschungen am Odenwaldlimes. Limesforschungen 12, 1973, Anm. 278-280: F. REUTTI: Tonverarbeitende Industrie im römischen Rheinzabern. Germania 61 (1989) 44.

${ }^{39}$ In Pannonien fanden neben Lezoux vor allem die Töpfer aus Martres-de-Veyre einen Abnehmerkreis. Vgl. I. MikL-CuRK: Terra sigillata in sorodne vrste keramike iz Poetovija (Terra Sigillata und ähnliche Keramikgattungen aus Poetovio). Dissertationes 9. Ljubljana 1969, 11; D. GABLER: Sigillaten auf dem Gebiet des Palatiums von Gorsium. Alba Regia 13 (1972 [1974]) 52; D. GABLER: Die Sigillaten vom Gebiete der Hercules-Villa in Aquincum. ActaArchHung 28 (1976) 53; D. GABleR-M. PichlerovÁ: Die Sigillaten von Gerulata. In: K. Kuzmová-J. Rajtár (Hrsg.): Gerulata I. Arch. Inst. der Slowakischen Akademie. Nitra 1996, Kat. 39.
${ }^{40}$ GABLER 1987,84

${ }^{41}$ D. Gabler-É. Kocztur: Terra Sigillata-Depotfund aus Gorsium. Alba Regia 15 (1976) 65-81.

${ }^{42}$ D. GABLER: Anteil der Sigillaten in Pannonien. Römisches Österreich 17-18 (1989-1990 [1991]) 93.

${ }^{43}$ Gy. JuHÁsz: A lezoux-i gyárak aquincumi lerakata (Die Aquincumer Niederlassung der Werkstätte von Lezoux). Archaeologiai Értesítő 41 (1936) 48.

${ }^{44}$ L. BARKóCZI-É. BóNIS: Das frührömische Lager und die Wohnsiedlung von Adony (Vetus Salina). ActaArchHung 4 (1954) $153-154$.

${ }^{45}$ D. GABLER: The Roman Fort Ács-Vaspuszta (Hungary) on the Danubian limes. BAR Int. Ser. 531, (Oxford 1989) 57-127; über die Rheinzaberner Sigillaten s. 456-464.

${ }^{46}$ GABLER 1987, 76-77.

${ }^{47}$ TH. FischeR: Das Umland des römischen Regensburg. Münchner Beitr. zur Vor- und Frühgeschichte 42 (1990) 44; A. FABER Das römische Auxiliarkastell und der Vicus von Regensburg-Kumpfmühl. Veröffentlichung der Kommission zur archäologischen Erforschung des spätrömischen Raetien. München 1994, 185. 
Die Rheinzaberner Sigillaten waren also bereits in der antoninischen Zeit konkurrenzfähig, obwohl sie damals neben den mittelgallischen Manufakturen in der Warenbelieferung nur eine untergeordnete Rolle spielten. ${ }^{48}$

Vergleicht man den - teils aufgrund der Obigen chronologisch bestimmten - frühen Rheinzaberner Bestand der einzelnen Fundorte mit den antoninischen Sigillaten von Lezoux, dann zeichnet sich das nachstehende Bild ab. ${ }^{49}$ Dieses Bild zeigt deutlich, dass die rheinische Ware zwischen 160 und 180 noch eine untergeordnete Rolle spielte. ${ }^{50}$

Die meisten Gefäße vertreten die mittlere Periode der Rheinzaberner Produktion in den Donauprovinzen (Gruppe des Comitialis, BfAttoni, Mammilianus, Ware mit E. 25/26, Helenius, Reginus II). ${ }^{51}$ Neulich kann die Ware des Janu II, die wieder früher datiert und dadurch die Verbindung mit Janu I deutlich gemacht wurde ${ }^{52}$ in die Zeitspanne zwischen 180 und 220/230 n. Chr. eingereiht werden. Auch diese Ware tritt in Pannonien sehr oft auf. Im Vergleich mit der Menge der frühen Rheinzaberner Ware ist die sprunghafte Zunahme der sog. mittleren Gruppe zu beobachten (Abb. 6).

\begin{tabular}{|l|c|c|}
\hline & $\begin{array}{c}\text { Frühe Produktionszeit } \\
\mathbf{( 1 5 0 / 1 5 5 - 1 7 0 / 1 7 8 ~ n . ~ C h r . )}\end{array}$ & $\begin{array}{c}\text { Mittlere Produktionszeit } \\
\text { (178-220/230 n. Chr.) }\end{array}$ \\
\hline Vindobona & 148 & 300 \\
\hline Wien-Michaelerplatz & $23,3 \%$ & $61,7 \%$ \\
\hline Wien-Rennweg 44 & 14 & 16 \\
\hline Carnuntum & 35 & 34 \\
\hline Carnuntum-Pfaffenberg & 7 & 28 \\
\hline Strebersdorf & 10 & 26 \\
\hline Gerulata & 13 & 99 \\
\hline Brigetio & 43 & 46 \\
\hline Baláca & 10 & 78 \\
\hline Aquincum-Zivilstadt & 21 & 54 \\
\hline Aquincum-Canabae & 12 & 73 \\
\hline Budaörs & 14 & $80 \%$ \\
\hline Páty & $20 \%$ & 33 \\
\hline Gorsium & 17 & \\
\hline
\end{tabular}

Die Belieferung von Rheinzabern war also in der spätantoninisch-severischen Zeit am stärksten. ${ }^{53}$ Wie es zu sehen ist, wuchs die Anzahl der Stücke, die sicher an eine Serie geknüpft werden können, beinahe auf jedem Fundort auf das Doppelte. Dazu kommt natürlich auch der Umstand, dass die mittlere Periode von Rheinzabern eine etwas längere Zeit in sich fasst als die frühe.

Die starke quantitative Zunahme der Importkeramik ist dadurch zu erklären, dass die wirtschaftliche Blütezeit der Donauprovinzen eben in diese Zeitspanne, in die Zeit der severischen Konjunktur, zu datieren ist. ${ }^{54}$ Damals waren die Sigillaten keine Luxuswaren mehr, sondern für die Haushalte erreichbare Artikel, die auch noch in die fortlebenden früheren einheimischen Siedlungen gelangen konnten. Die Gründung der Töpferei von Westerndorf kann eher mit dem zur severischen Zeit wachsenden Wohlstand der Donauprovinzen zusammenhängen, mit ihrer

${ }^{48}$ Zur Gruppierung s. M. GIMBER: Anmerkungen zur Fortsetzung der Diskussion um die Chronologie der Rheinzaberner Relieftöpfer. BVbl 64 (1999) 381-392.

${ }^{49}$ GABLER 1987, 84.

${ }^{50}$ Über eine längere Nutzungszeit der Waren des Janu I und Reginus I s. V. SElKE: Römische Funde aus Dambach. Materialhefte zur Bayrischen Archäologie 100. Kallmünz/Opf. 2014, 122; A. Schaub: Markomannenkriegszeitliche Zerstörungen in Sulz am Neckar - Ein tradierter Irrtum. Bemerkungen zu reliefverzierter Terra Sigillata vom Ende des zweiten Jahrhunderts. Markomannenkriege Ursachen und Wirkungen. Hrsg. von H. Friesinger, J. Tejral und A. Stuppner. Brno 1994, 440.

${ }^{51}$ MeEs 2002, 330-331 (BfAttoni), 338-339 (Mammilianus), 338 (Ware mit E. 25/26), 343 (Reginus II), 346 (Helenius), 350-352 (Primitivus).
${ }^{52}$ M. GIMBER: Eine Reliefschüssel des Janus II-Ateliers mit Randstempel. Im Dienste Roms. Festschrift für H. U. Nuber. Remshalden 2006, 375; K. BitTNER: Zur Fortsetzung der Diskussion um die Chronologie der Rheinzaberner Relieftöpfer. BVbl 61 (1996) 144.

${ }^{53}$ Z. HANTOS: A rheinzaberni reliefdíszes sigillaták pannoniai forgalma (Über den Verkehr reliefverzierter Terra Sigillaten aus Rheinzabern in Pannonien). Archaeologiai Értesítő 130 (2005) 105-135.

${ }^{54}$ A. Mócsy: Pannonia and Upper Moesia. A History of the Middle Danubian Provinces of the Roman Empire. London-Boston 1974, 218, besonders 232, 236-237; A. Mócsy: Pannonien und die Soldatenkaiser. ANRW II (1977) 571-579; L. BARKÓCZI: The population of Pannonia from Marcus Aurelius to Diocletian. ActaArchHung 16 (1964) 296; D. GABLER: Munera Pannonica. Archaeologiai Értesítő 93 (1966) 20 ff.; L. BALLA-T. BuOCZ-Z. KÁDÁR-A. MócsY-T. SZENTLÉLEKY: Die römischen Steindenkmäler von Savaria. Budapest 1971, $29 \mathrm{ff}$.

Acta Archaeologica Academiae Scientiarum Hungaricae 67, 2016 


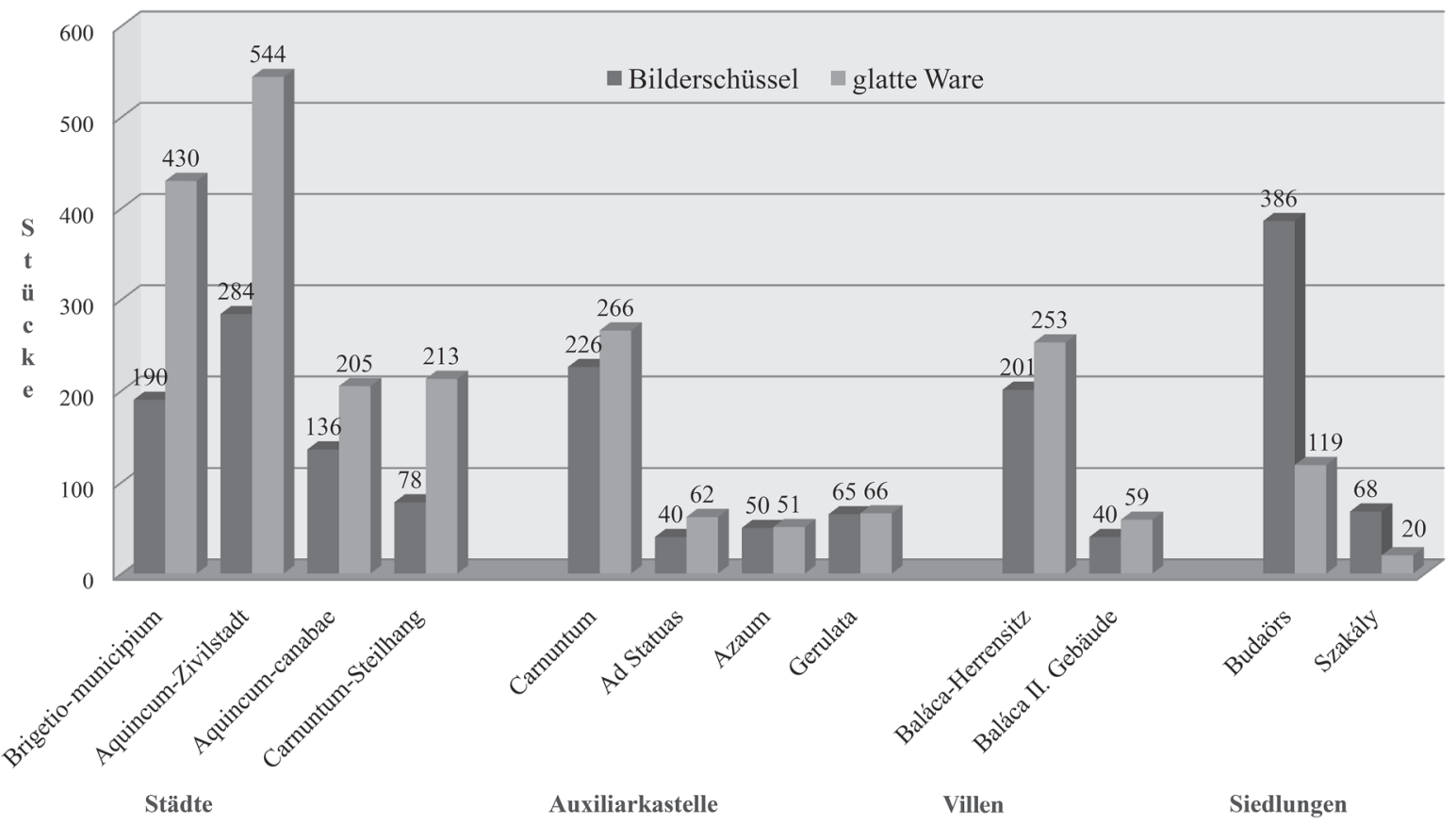

Abb. 5. Anteil der Bilderschüsseln und der glatten Ware in verschiednen Fundorten Pannoniens

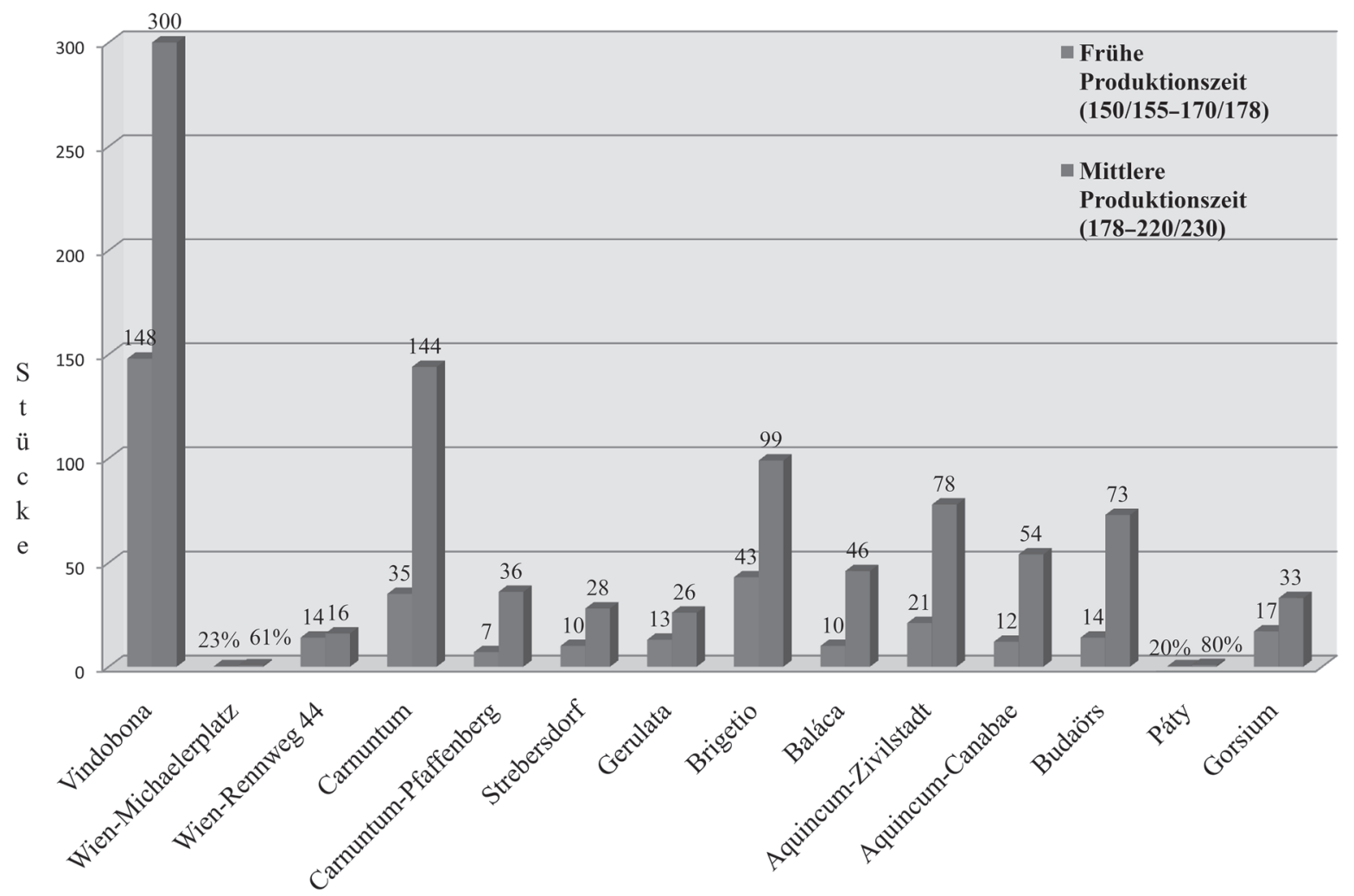

Abb. 6. Anteil der antoninischen und der severischen Sigillaten in verschiedenen Fundorten Pannoniens 
stabilen Wirtschaftslage und der sich aus der Konjunktur ergebenden Import-Nachfrage einer Situation, die möglich machte, dass sich außer Rheinzabern auch andere Manufakturen in die Versorgung der Provinz einschalten konnten. Der Import aus Rheinzabern und Westerndorf strömte gleichzeitig nach Pannonien. ${ }^{55}$ Ein Vergleich des Anteils beider Töpferzentren auf verschiedenen Fundorten zeigt, dass der Anteil von Westerndorf in der Grenzzone hoch ist; die Städte und Siedlungen am Limes weisen neben der rheinischen Manufaktur in größerer Menge Westerndorfer Ware auf. Das Provinzinnere wurde in der Severerzeit weiterhin aus Rheinzabern mit Sigillaten versorgt.

Über das Ende der Rheinzaberner Reliefsigillata-Produktion stehen nur wenige Angaben zur Verfügung. Nur in Niederbieber und Saalburg weisen die Münzen auf eine bis 259/260 n. Chr. andauernde ununterbrochene Besiedlung eindeutig hin. ${ }^{56}$ Andererseits wurde es klar, dass das Datum 259/260 nicht mehr für einen Zeitpunkt, mit dem man die totale Zerstörung des obergermanisch-raetischen Limes in Verbindung bringen könnte, gehalten werden kann. $^{57}$

Die drastische Verminderung des Importes nach 233/242 n. Chr. kann mit der Stagnierung der Handelsbeziehungen, der Minderung der Kaufkraft und dem dramatischen Verfall des Lebensniveaus der städtischen Bevölkerung in der Grenzzone in Zusammenhang gebracht werden. ${ }^{58}$ Das spiegelt natürlich die außen- und innenpolitische Lage des Reichs in den 60er Jahren des 3. Jahrhunderts wider.

ANHANG

\begin{tabular}{|c|c|}
\hline $\begin{array}{c}\text { Fundorte der Rheinzaberner Sigillaten } \\
\text { seit dem Erscheinen Gabler } 1987\end{array}$ & Literatur \\
\hline 1. Biedermannsdorf & D. TALAA-I. HERMANN, FÖ 44 (2005) 528-529 \\
\hline 2. Moosbrunn & J. MATOUSCHEK-H. NOWAK, FÖ 40 (2001) 658 \\
\hline 3. Sommerein & J. MATOUSCHEK-H. NOWAK, FÖ 40 (2001) 664 \\
\hline 4. Wangheim & J. MATOUSCHEK-H. NOWAK, FÖ 40 (2001) 666 \\
\hline 5. Kaisersteinbruch & J. MATOUSCHEK-H. NowAK, FÖ 40 (2001) 630 \\
\hline 6. Potzneusiedl & CH. FARKA-H. NOWAK, FÖ 24-25 (1985-1986) 277 \\
\hline 7. Gattendorf & CH. FARKA-J. KNOPF, FÖ 26 (1987) 229 \\
\hline 8. Kittsee & J. MATOUSCHEK-H. NowAK, FÖ 38 (1999) 817 \\
\hline 9. Deutsch Jahndorf & J. MATOUSCHEK-H. NOWAK, FÖ 40 (2001) 620 \\
\hline 10. Zurndorf & E. GRILL-H. NOWAK, FÖ 24-25 (1985-1986) 279 \\
\hline 11. Nickelsdorf & J. MATOUSCHEK-H. NowAK, FÖ 40 (2001) 633 \\
\hline 12. Leithaprodersdorf & J. MATOUSCHEK-H. NOWAK, FÖ 40 (2001) 630 \\
\hline 13. Pöttsching & J. MATOUSCHEK-H. NOWAK, FÖ 38 (1999) 828 \\
\hline 14. Wulkaprodersdorf & J. MATOUSCHEK-H. NOWAK, FÖ 38 (1999) 829 \\
\hline 15. Purbach & J. MATOUSCHEK-H. NOWAK, FÖ 40 (2001) 636 \\
\hline 16. Podersdorf am See & J. MATOUSCHEK-H. NOWAK, FÖ 40 (2001) 635 \\
\hline 17. Halbturn & J. MATOUSCHEK-H. NOWAK, FÖ 40 (2001) 622 \\
\hline 18. Antau & J. MATOUSCHEK-H. NOWAK, FÖ 40 (2001) 616 \\
\hline 19. St. Margarethen & Сh. FARKA-J. MatouscheK, FÖ 28 (1989) 209 \\
\hline 20. Illmitz & $\begin{array}{l}\text { J. MATOUSCHEK-H. NOWAK, FÖ } 42 \text { (2003) } 695 \\
\text { E. GRILL-H. NOWAK, FÖ } 32 \text { (1993) } 722\end{array}$ \\
\hline 21. St. Andrä am Zicksee & CH. FARKA-H. NOWAK, FÖ 27 (1988) 300 \\
\hline 22. Mosonszentpéter & $\begin{array}{l}\text { R. PuszTAI: Római kori településmaradvány, villa rustica Mosonszentpéter község keleti } \\
\text { határában, a Kecske földön. Arrabona } 39 \text { (2001) 26, Abb. } 10.1\end{array}$ \\
\hline 23. Neudörfl & J. MATOUSCHEK-H. NOWAK, FÖ 40 (2001) 633 \\
\hline 24. Marz & СH. FARKA-H. NOWAK, FÖ 27 (1988) 297, FÖ 38 (1999) 818 \\
\hline
\end{tabular}

${ }^{55}$ D. GABLER: Terra sigillaták Aquincum legkorábbi táborából és annak helyén emelt későbbi római épületekből (Samian ware from the earliest fort of Aquincum). Budapest Régiségei 45 (2012) 127.

${ }^{56}$ MeEs 2002, 101.

${ }^{57}$ K. STRoBel: Pseudophänomene der römischen Militärund Provinzgeschichte der Jahrzehnte nach 253 n. Chr. am Rhein und der oberen Donau. In: N. Gudea (ed.): Roman Frontier Studies. Proceedings of the 17th International Congress of Roman Frontier Studies. Zălau 1999, 9-30.

${ }^{58}$ Man kann vielleicht auch eine Änderung der Tischsitten vermuten; s. SELKE 2014, 123-124. 


\begin{tabular}{|c|c|}
\hline $\begin{array}{c}\text { Fundorte der Rheinzaberner Sigillaten } \\
\text { seit dem Erscheinen Gabler } 1987\end{array}$ & Literatur \\
\hline 25. Großhöflein & J. MATOUSCHEK-H. NOWAK, FÖ 40 (2001) 622 \\
\hline 26. Klingenbach & J. MATOUSCHEK-H. NOWAK, FÖ 40 (2001) 630 \\
\hline 27. Neckenmarkt & $\begin{array}{l}\text { W. KRAFT, FÖ 24-25 (1985-1986) } 274 \\
\text { CH. FARKA-V. SEYFRIED, FÖ 24-25 (1985-1986) } 275 \\
\text { J. MATOUSCHEK-H. NowAK, FÖ } 38 \text { (1999) 820 }\end{array}$ \\
\hline 28. Strebersdorf & J. MATOUSCHEK-H. NOWAK, FÖ 37 (1998) 760-764 \\
\hline 29. Girm & J. MATOUSCHEK-H. NOWAK, FÖ 40 (2001) 621 \\
\hline 30. Klostermarienberg & J. MATOUSCHEK-H. NOWAK, FÖ 40 (2001) 630 \\
\hline 31. Kemenesszentpéter & Grabung von S. Palágyi im Jahr 1990 \\
\hline 32. Visegrád-Lepence & Grabung von P. Gróf im Jahr 2009 \\
\hline 33. Budaörs & Római vicus Budaörsön (hrsg. von K. Ottományi), Budapest 2012, 422-435 \\
\hline 34. Érd & Grabung von K. Ottományi im Jahr 2003 \\
\hline 35. Bakonyszentlászló (?) & CSIRKE-GABLER-PALÁGYI 2006, 27-32 \\
\hline 36. Ugod & Grabung von S. Palágyi, Laczkó Dezső Múzeum, Inv.-Nr. 69.18.48 \\
\hline 37. Veszprémvarsány & CSIRKE-GABLER-PALÁGYI 2006, 204 \\
\hline 38. Csetény & CSIRKE-GABLER-PALÁGYI 2006, 171 \\
\hline 39. Szápár & CSIRKE-GABLER-PALÁGYi 2006, 188-189 \\
\hline 40. Zirc & CSIRKE-GABLER-PALÁGYI 2006, 204 \\
\hline 41. Bakonynána & CSIRKE-GABLER-PALÁGYI 2006, 27 \\
\hline 42. Várpalota & CSIRKE-GABLER-PALÁGYI 2006, 201 \\
\hline 43. Pátka & PALÁGYI-NAGY 2002, 93 \\
\hline 44. Kádárta & CSIRKE-GABLER-PALÁGYI 2006, 178 \\
\hline 45. Litér & CSIRKE-GABLER-PALÁGYI 2006, 181 \\
\hline 46. Berhida & CSIRKE-GABLER-PALÁGYI 2006, 169 \\
\hline 47. Ősi & CSIRKE-GABLER-PALÁGYI 2006, 186 \\
\hline 48. Balatonfőkajár & CSIRKE-GABLER-PALÁGYI 2006, 35 \\
\hline 49. Lovas & CSIRKE-GABLER-PALÁGYI 2006, 181 \\
\hline 50. Balatonalmádi & CSIRKE-GABLER-PALÁGYI 2006, 33 \\
\hline 51. Balatonfüred & CSIRKE-GABLER-PALÁGYI 2006, 35 \\
\hline 52. Paloznak & CSIRKE-GABLER-PALÁGYI 2006, 187 \\
\hline 53. Nagyvázsony & CSIRKE-GABLER-PALÁGYI 2006, 183 \\
\hline 54. Örvényes & CSIRKE-GABLER-PALÁGYI 2006, 185 \\
\hline 55. Balatonakali & CSIRKE-GABLER-PALÁGYI 2006, 33 \\
\hline 56. Szentgál & CSIRKE-GABLER-PALÁGYI 2006, 189 \\
\hline 57. St. Martin an der Raab & S. LAMM, FÖ 45 (2006) 397 \\
\hline 58. Balatonőszöd & Grabung von G. Serlegi im Jahr 2002 \\
\hline 59. Söjtör & PALÁGYI-NAGY 2002, 93 \\
\hline 60. Gelsesziget & PaLÁGYI-NAGY 2002, 93 \\
\hline 61. Dunaszentgyörgy & Grabung im Jahr 2011 \\
\hline 62. Kisvaszar & $\begin{array}{l}\text { P. PROHÁSZKA: Császárkori halomsíros temető Kisvaszar határában (Baranya m.) (Das kaiser- } \\
\text { zeitliche Hügelgräberfeld in der Gemarkung von Kisvaszar). Folia Arch. } 55 \text { (2011-2013) 82, } 87\end{array}$ \\
\hline 63. Cserdi & $\begin{array}{l}\text { M. SZABÓ-A. KISS-R. MOLNÁR-B. NAGY-R. NEMÉNYI-A. SZABÓ-P. N. TAKÁCS-I. TALABÉR: } \\
\text { Előzetes jelentés a Cserdi község (Baranya m.) melletti római villagazdaság 2012. évi próbafel- } \\
\text { tárásáról és terepi kutatásokról (Roman villa at Cserdi (Baranya county) - Preliminary report on } \\
\text { archaeological investigations conducted in 2012). FIRKÁK III. Fiatal római koros kutatók } 3 . \\
\text { konferenciakötete. Szombathely 2014, 274-275, Pl. II }\end{array}$ \\
\hline 64. Orešac & $\begin{array}{l}\text { R. MAKJANIĆ: Sigillata from Orešac, Vincek and Radijevac collections. Prilozi odjela za arheo- } \\
\text { logiju } 7 \text { (1990) 31-44, bes. 35-37 }\end{array}$ \\
\hline 65. Babarc & Zs. VISY-F. HoRVÁTH, Specimina nova 8 (1992 [1994]), 125 \\
\hline
\end{tabular}


CSIRKE-GABLER-PALÁGYi 2006

GABLER 1987

MEES 2002

PALÁGYI-NAGY 2002

SELKE 2014

WEBER-HIDEN 1996

\section{ABGEKÜRZT ZITIERTE LITERATUR}

= O. CsiRke-D. GABlER-S. PALÁGYi: Terra sigillata edények a veszprémi Laczkó Dezső Múzeum gyüjteményében (Terra Sigillata-Gefäße im Museum „Laczkó Dezső“ in Veszprém). Veszprém 2006.

= D. GABLER: Einige Besonderheiten der Verbreitung der Rheinzaberner Sigillaten in Pannonien. Bayerische Vorgeschichtsblätter 52 (1987) 75-104.

= A. MEEs: Organisationsformen römischer Töpfermanufakturen am Beispiel von Arezzo und Rheinzabern mit Berücksichtigung von Papyri, Inschriften und Rechtsquellen. Röm.-Germ. Zentralmuseum Monographien. Mainz 2002.

= S. K. PALÁGYI-L. NAGY: Römerzeitliche Hügelgräber in Transdanubien (Ungarn). Budapest 2002.

= V. SELKE: Römische Funde aus Dambach. Materialhefte zur Bayrischen Archäologie 100. Kallmünz/ Opf. 2014.

= I. WEBER-HIDEN: Die reliefverzierte Terra Sigillata aus Vindobona. Teil 1. Legionslager und Canabae. Wiener Arch. Studien 1. Wien 1996. 